\title{
Interpretación y medición comparada de líneas granulométricas continuas
}

PEDro enrique Grinszpan, Profesor titular de la E. Otto Krause e I.T.S. (*)

IV. REUNION TECNICA SOBRE TECNOLOGIA DEL HORMIGON/A.A.T.H.

Mendoza - Noviembre 4 al 10 de 1979

\section{R E S U M E N}

Se propone en este trabajo una expresión analítica que vincula al módulo de finura de Abrams con el tamaño límite superior de la serie granulométrica y con el grado de una función parabólica identificable con la analizada a efectos de poder localizar la composición con relación a los dominios de conveniencia establecidos por las normas y de este modo realizar una medición comparada de su aptitud.

Se introduce el concepto de coeficiente de aptitud a efectos de medir en términos relativos la bondad de una composición granulométrica continua y se propone una escala de clasificación y calificación, cuya inclusión en los pliegos de especificaciones se recomienda para la recepción de los agregados.

Por último, la función adoptada, que se considera una generalización de la distribución de Gessner, es comparada con las de Bolomey y Ross, estableciéndose la diferencia con éstas.

\section{INTRODUCCION}

\subsection{Antecedentes}

En el desarrollo de la Tecnología del Hormigón hubo numerosos intentos para expresar las composiciones granulométricas mediante expresiones analíticas que representasen leyes de comportamiento óptimo al ser aplicadas en la realización de hormigones. En tal sentido puede enumerarse a título de ejemplo, las siguientes:

a) La regla lineal antigua;

b) El método de Fuller-Thompson;

c) La variante de Gessner sobre la anterior;

d) Las funciones de Bolomey;

e) La función de Ross (EMPA);

f) El criterio de Furnas;

(*) Profesor de Construcciones de Hormigón Armado, Estática y Estabilidad de las Construcciones, Proyectos de Hormigón Armado, y Tecnologia del Hormigón y Práctica de Obra, de los cursos regulares y para técnicos graduados. 
g) El criterio de Valette;

h) Los estudios de Caquot;

i) El método de Faury; y

j) El método de Joisel.

Las cinco primeras formulan propuestas que conducen a la obtención de líneas granulométricas continuas, debiendo recordarse que la segunda y la cuarta corresponden a composiciones que incluyen el cemento. La sexta y la séptima son de limitada aplicación basadas en composiciones de tamaños discontinuos. Las tres últimas (octava a décima) son consecuencia de los importantes estudios de Caquot sobre la relación entre la proporción de huecos y el tamaño de las partículas, y el efecto pared vinculado a su vez con el llamado radio medio (de encofrado) de la zona más densamente armada; estas formulaciones conducen a métodos sensiblemente complejos.

Por otra parte se tiene el método debido a Abrams de evaluar la composición granulométrica por medio de un índice numérico global vinculado a las series americanas de tamices de Tyler y A.S.T.M. Este criterio ha permitido formular el concepto de composiciones granulométricas equivalentes para aquéllas de igual módulo de finura, pudiendo así vincularse inclusive las composiciones continuas con aquellas que no lo son, en cuanto a su bondad para el empleo de las mismas. El empleo de este módulo permite, así mismo, analizar compensaciones y correcciones en las composiciones granulométricas.

\subsection{Expresiones analíticas}

Consideraremos la expresión de la ordenada de la línea granulométrica continua (entre la curva y el lado inferior del gráfico) cuyo significado es el porcentaje total del material "Pasante" por debajo de un tamiz; su complemento es el porcentaje total del material "Retenido" por encima del mismo tamiz; la suma de ambos valores corresponde al cien por ciento del material. Utilizaremos las siguientes designaciones:

$d_{i}=$ paso de malla del tamiz " $i$ " de la serie normalizada A.S.T.M. (E.U. de N.A.) que cumple la siguiente relación:

$$
2 \cdot d_{i-1}=d_{i}=1 / 2 \cdot d_{i+1}
$$

constituida por los tamices (en orden creciente): n.200;n.100; n.50; n.30;n.16; n.8; n.4; $3 / 8 "$; $3 / 4 "$; $3 / 2 "$; 3 "; 6" y 12 ", que se identifican con los tamaños: $d_{0} ; d_{1} ; d_{2} ; \ldots d_{\mathrm{i}} ; \ldots d_{\mathrm{n}}=D$ correspondiente al tamaño límite superior de la composición y donde los subíndices numeran en orden creciente los tamices de la serie mencionada: $O ; \ldots i ; \ldots N$.

$d_{0}=$ tamaño inferior no considerado para el módulo de finura de Abrams.

$d_{\mathrm{n}}=D=$ tamaño límite superior de la composición estudiada, no el de la serie de tamices con $P_{\mathrm{n}}=100 \%$.

$P_{\mathrm{i}}=$ porcentaje de material depositado en el tamiz $d_{\mathrm{i}}$ proveniente de los superiores igual al retenido parcial en dicho tamiz.

$P_{\mathrm{i}}=$ porcentaje total de material que pasa por el tamiz $d_{\mathrm{i}}$.

$R_{\mathrm{i}}=$ porcentaje total del material que no pasa por el tamiz $d_{\mathrm{i}}$ quedando en éste y en los superiores.

$r=$ exponente.

$m=m_{\mathrm{f}}=$ módulo de finura de Abrams $=$ (Sum. $\left.R_{\mathrm{i}}\right)_{1, \mathrm{n}} / 100$.

$b=$ base de la progresión geométrica, para la serie A.S.T.M. resulta $b=2$.

Sum $=S=$ sumatoria. 


\subsection{Composiciones continuas}

La más antigua, conocida como regla lineal se puede expresar del siguiente modo:

a)

$$
\% P_{\mathrm{i}} / 100=d_{\mathrm{i}} / d_{\mathrm{r}}=d_{\mathrm{i}} / D
$$

La función elíptica-lineal de Fuller-Thompson (b) queda sustituida por la variante parabólica de Gessner:

c)

$$
\% P_{\mathrm{i}} / 100=\left(d_{\mathrm{i}} / D\right)^{\mathrm{r}}=\left(d_{\mathrm{i}} / d_{\mathrm{n}}\right)^{\mathrm{r}} ; \operatorname{con} r=1 / 2
$$

de la cual la primera es un caso particular para $(r=1 / 2$; parábola cuadrática) $r=1$ (recta).

Las funciones de Bolomey para la curva continua de "cemento y agregado" según la siguiente expresión:

$$
\% P_{\mathrm{i}} / 100=(1-B)+B \cdot\left(d_{\mathrm{i}} / D\right)^{\mathrm{r}} ; \operatorname{con} r=1 / 2
$$

en la cual el coeficiente " $B$ " adopta los siguientes valores en función de la clase de agregado (redondeado o angular) y de la consistencia de la mezcla fresca (densa/plástica/o/ /fluida):

haciendo $B=1-B^{\circ}$ y siendo " $B^{\circ}$ " igual a:

DENSA

PLAstica

FLUIDA

AGR. REDOND.

0,08

0,10

0,12

AGREG. ANG.

0,10

0,12

0,15

luego es posible expresar el coeficiente " $B$ " de ajuste, de la siguiente forma:

$$
B=1-(0,10) \cdot f \cdot k
$$

en la cual " $f$ " $=0,8 / 1,0 / 1,2$ según la consistencia y " $k$ " $=1,0 / 1,2$ según la clase de agregado.

Por último, la función compuesta de Ross, que se puede exponer de la siguiente manera:

$$
\% P_{\mathrm{i}} / 100=1 / 2 \cdot\left[d_{\mathrm{i}} / D+\left(d_{\mathrm{i}} / D\right)^{\mathrm{r}}\right]
$$

en la cual tendremos también $r=1 / 2$.

\subsection{Módulo granulométrico}

La expresión general válida para vincular al módulo granulométrico correspondiente a un tamaño " $d$ " dado con un tamaño inferior de la serie de tamices considerada " $d_{0}$ " y la base de la progresión geométrica " $b$ " que relaciona los tamaños de dicha serie es:

$$
d=d_{0} \cdot b^{\mathrm{m}}
$$


donde " $m$ " es el módulo, aplicando logaritmos se tiene:

$$
\log d=\log d_{o}+m \cdot \log b
$$

donde en el caso del módulo decádico " $m_{\mathrm{d}}$ " de Spindel resulta con $d_{0}=1$ micron, $b=10$, siendo entonces el módulo igual a la abscisa del tamaño considerado en la escala logarítmica:

$$
\log d=0+m_{\mathrm{d}} \cdot 1=m_{\mathrm{d}}
$$

en tanto que para el módulo de finura " $m_{\mathrm{f}}$ " de Abrams, resulta con $d_{0}=741 / 2$ micro$\mathrm{n} \in \mathrm{s}, b=2$ la siguiente forma:

$$
(\log d-1,872) / 0,301=m_{\mathrm{f}}
$$

de la cual resulta que el módulo de Abrams equivale al número de orden, en sentido creciente, de los tamices de la serie normalizada A.S.T.M. para "O" correspondiente a $d_{0}$, " $i$ " para $d_{\mathrm{i}}, \mathrm{y}$ " $n$ " para $d_{\mathrm{n}}=D$ (límite supe rior considerado).

Ambos módulos se vinculan mediante la ecuación siguiente:

$$
\text { " } m_{\mathrm{d}} "-1,872=0,301 \cdot \text { " } m_{\mathrm{f}} "
$$

en la que resulta:

$$
" m_{\mathrm{f}} "=\left(\operatorname{Sum} R_{\mathrm{i}}\right)_{1, \mathrm{n}} / 100=A_{\mathrm{H}} \cdot(30,1)
$$

Siendo $A_{\mathrm{H}}$ el área de Hummel, superficie delimitada por la línea granulométrica, la vertical por $d_{0}$, y la horizontal correspondiente a $P=100 \%\left(p / d_{\mathrm{n}}=D\right)$.

\section{FUNCION CONTINUA GENERALIZADA}

\subsection{La parábola generalizada de grado " $r$ "}

Si retomamos la ecuación (II) tendremos que:

$$
\begin{gathered}
\% P / 100=\left(d_{\mathrm{i}} / d_{\mathrm{n}}\right)^{\mathrm{r}}=\left(b^{\mathrm{i}} \cdot d_{0}\right)^{\mathrm{r}} /\left(b^{\mathrm{n}} \cdot d_{0}\right)^{\mathrm{r}}=b^{\mathrm{i} r} / b^{\mathrm{n} r}= \\
=b^{[\mathrm{r}(\mathrm{i}-\mathrm{n})]}=\left(b^{\mathrm{r}}\right)^{(\mathrm{i}-\mathrm{n})}=a^{(\mathrm{i}-\mathrm{n})}
\end{gathered}
$$

\subsection{Las líneas de tamizado de la norma DIN 1045}

Si se compara la expresión (VI) con los límites que establece esta norma entre las zonas "3.RECOMENDABLE", "4.ACEPTABLE", y las "1 y 5.INADECUADAS" para distintos tamaños límites superiores " $D$ ", puede notarse que (ver fig. 1) las tres líneas $A-B-C$ corresponden con suficiente aproximación a parábolas de distinto grado " $r$ " tal que:

Línea " $A$ " (lím. entre 1 y 3), exponente " $r "=0,666 \ldots$

Línea " $B$ " (lím. entre 3 y 4), exponente " $r$ " =0,333 $\ldots$

Línea "C" (lím. entre 4 y 5), exponente " $r "=0,222 \ldots$ 
Además por el centro de la zona "3.RECOMENDABLE" pasa en forma equidistante una línea coincidente con la parábola de grado " $r$ " $=0,500$ propuesta por GESSNER.

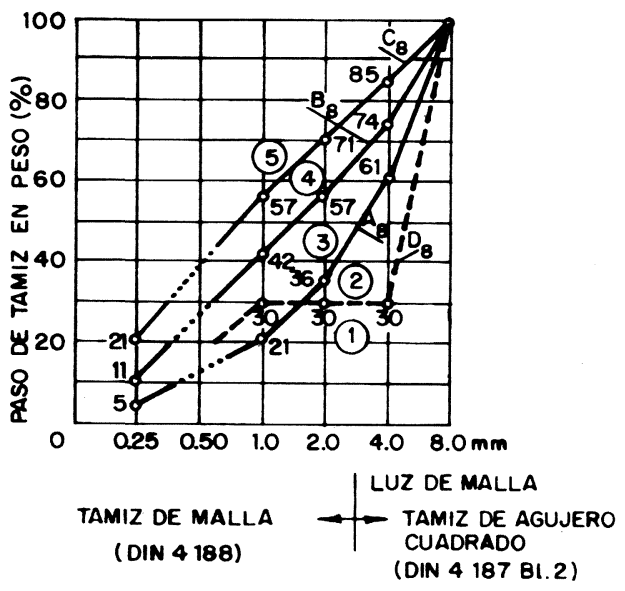

Linea de tamiz con un grano máximo de $8,0 \mathrm{~mm}$.

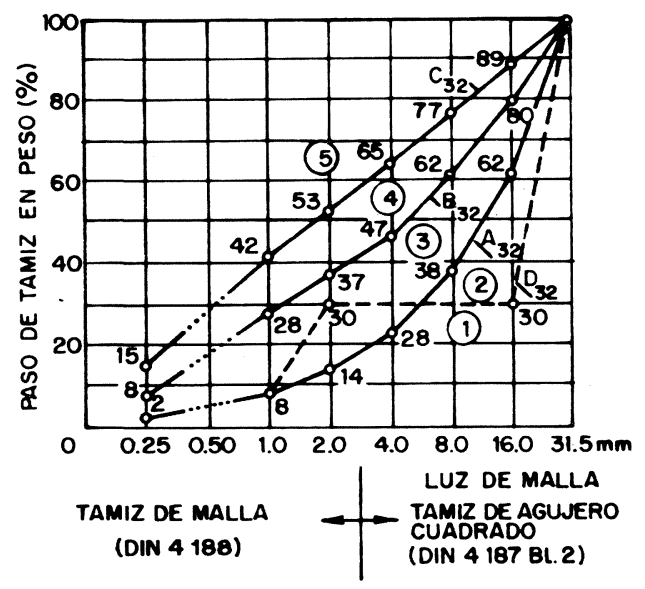

Línea de tamiz con un grano máximo de $\mathbf{3 2 , 0} \mathrm{mm}$.

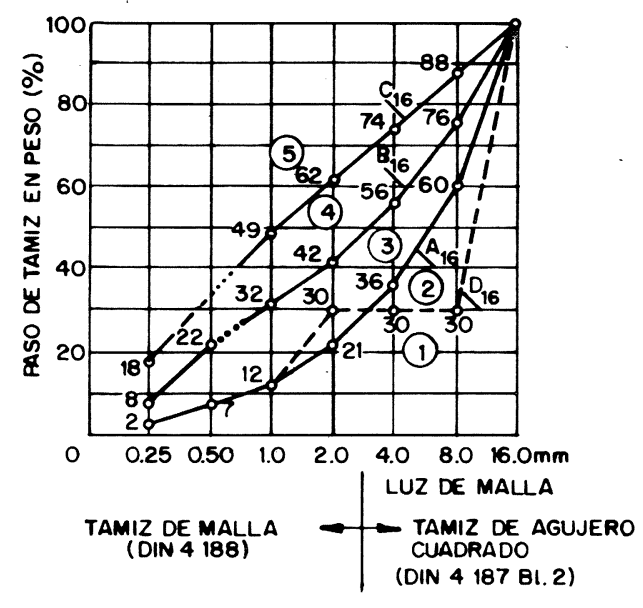

Línea de tamiz con un grano máximo de $16,0 \mathrm{~mm}$.

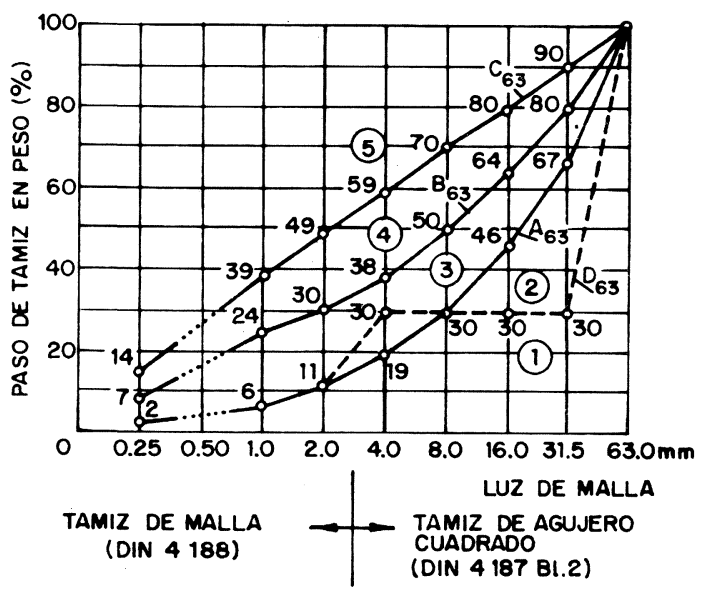

Linea de tamiz con un grano máximo de $63,0 \mathrm{~mm}$.

Fig. 1

En resumen, para líneas de exponente " $r$ " menor de 0,222 tendremos curvas INADECUADAS por excesivos finos situadas en la zona 5 por sobre la línea $C$, mientras que para un exponente " $r$ " superior a 0,666 se hallará por debajo de la línea $A$ en la zona 1-2 siendo INADECUADAS por excesivos gruesos. Para exponentes " $r$ " entre 0,222 y 0,333 se hallará en la zona "4.ACEPTABLE" y finalmente para exponentes " $r$ " entre 0,333 y 0,666 se ubicará en la zona "3.RECOMENDABLE" por cuyo medio pasa la parábola cuadrática de $r=0,5$ debida a Gessner, cuyas cualidades quedan así definidas.

Resulta evidente que el exponente " $r$ " es un elemento idóneo para caracterizar a una línea granulométrica continua y clasificar su aptitud en forma global. 


\subsection{El módulo de finura en la función generalizada}

En 2.1. se ha determinado la ordenada de la función, que resulta:

$$
\% P / 100=a^{(i-n)}
$$

pudiéndose a partir de este valor obtener la ordenada complementaria, que es el retenido acumulado:

$$
\% R / 100=1-\% P / 100=1-a^{(i-n)}
$$

luego, efectuando la sumatoria se obtiene el módulo de finura:

$$
\begin{array}{r}
m_{\mathrm{f}}=m=\operatorname{Sum}_{1, \mathrm{n}} \% R / 100=S_{1, \mathrm{n}}\left(1-a^{\mathrm{i}-\mathrm{n}}\right)= \\
=n-S_{1, \mathrm{n},} a^{\mathrm{i}-\mathrm{n}}=n-a^{-\mathrm{n}} \cdot S_{1, \mathrm{n}} a^{\mathrm{i}}
\end{array}
$$

siendo

$$
S_{1, \mathrm{n}}=\left(a^{\mathrm{n}+1}-a\right) /(a-1)
$$

y sustituyenda, se tiene:

$$
m=n-\frac{a^{\mathrm{n}+1}-a}{a^{\mathrm{n}+1}-a^{\mathrm{n}}}=n-\frac{1-1 / a^{\mathrm{n}}}{1-1 / a}
$$

y llamando

$$
A=1 / a
$$

queda finalmente:

$$
m=n-\left(1-A^{\mathrm{n}}\right) /(1-A)
$$

expresión que vincula al módulo de finura de Abrams con el tamaño límite $(D)$ de la composición considerada, mediante " $n$ ", y con el grado de la función $(r)$, por medio de $A=$ $=1 / a=1 / b^{r}$ (fig. 2).

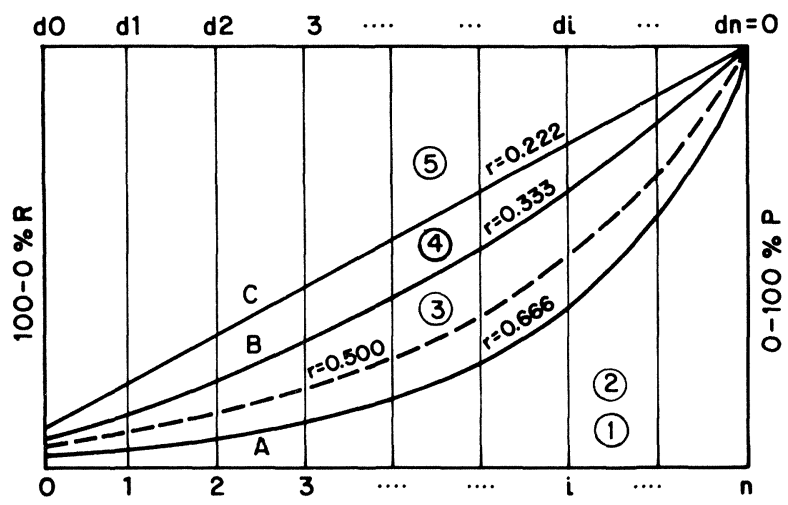

Fig. 2 


\subsubsection{Funciones con el origen desplazado}

En el caso de las composiciones continuas de gruesos solamente (ver fig. 3 ) pueden considerarse como si tuviesen el origen desplazado en el valor: " $d_{n}$ " $=d_{\mathrm{j}}-d_{0}$ y por consiguiente se extiende un intervalo que va del origen desplazado hasta $D=d n$, es decir (ver figura 4):

$$
n^{\prime}=d_{n}-d_{j}=n-d n
$$

resultando la expresión (IX) modificada en:

$$
m=\left(d n+n^{\prime}\right)-\left(1-A^{\mathrm{n}^{\prime}}\right) /(1-A)=n-\left(1-A^{\mathrm{n}^{\prime}}\right) /(1-A)
$$

es decir, que únicamente se modifica el exponente ( $\left.n^{\prime}\right)$.

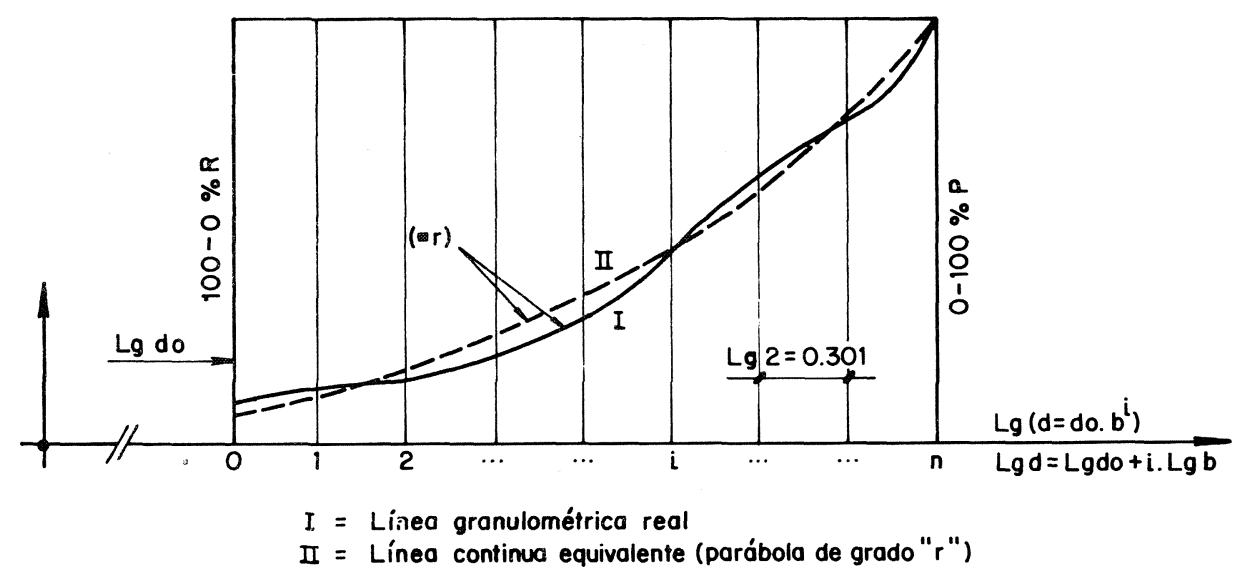

Fig. 3

\subsubsection{Determinación del grado " $r$ " de la función}

Llamando módulo complementario " $m c$ " $=n-m$ al complemento del área de Hummel (en la escala 1/30,1), diferencia entre " $n$ " correspondiente al tamaño límite superior y el módulo de finura " $m_{\mathrm{f}}$ " de Abrams, podemos transformar las expresiones anteriores (IX) $\mathrm{y}(\mathrm{X})$ en la siguiente:

$$
A=1-\left(1-A^{\mathrm{s}}\right) / m c \quad \text { con } \tilde{n}=n \text { ó } n^{\prime}
$$

ecuación que permite hallar " $A$ " mediante un proceso iterativo.

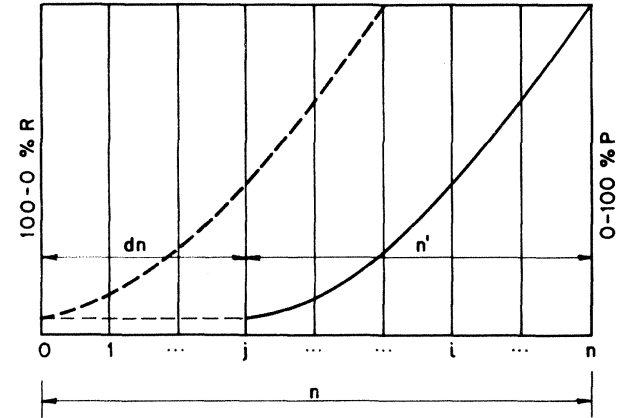

Fig. 4

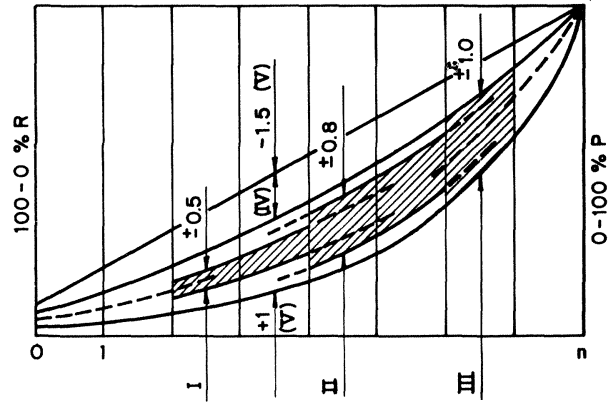

Fig. 5.-Dominio de los coeficientes de aptitud (CA). 
Hallado el valor de " $A$ " se obtiene inmediatamente:

$$
A=1 / a_{1}=1 / b^{r} \quad \ldots \quad r=\log (1 / A) / 0,301
$$

obtenido este último valor resulta inmediato localizar la ubicación de la línea granulométrica o en su defecto la línea continua equivalente, con relación a la zona de convenienoia de las normas (DIN, etc.).

\section{COEFICIENTE DE APTITUD}

3.1. Comparando la línea granulométrica de grado " $r$ " con la ideal de GESSNER (parábola para $r=0,500$ ) situada en el medio del intervalo:

$$
\begin{aligned}
\text { diferencia } " r " & =0,666-0,333=0,333 \\
\text { diferencia media } & =0,333 / 2=0,1666
\end{aligned}
$$

resulta posible relacionar la diferencia de la curva considerada con la diferencia media.

\subsection{Medición comparada}

Se puede de este modo establecer mediante dicha comparación el siguiente "coeficiente de aptitud":

COEFICIENTE APTITUD "CA" $=(r-0,500) / 0,1666=6 \cdot(r-0,5)$

Dándose para:

(ver figura 5)

$$
\begin{aligned}
& r=0,666 \quad C A=+1 \quad \text { (Línea A) } \\
& r=0,500 \quad C A=0 \quad \text { (Gessner) } \\
& r=0,333 \quad C A=-1 \quad \text { (Línea B) } \\
& r=0,222 \quad C A=-1,6 \quad \text { (Línea } C)
\end{aligned}
$$

Proponiéndose la siguiente escala de valoración:

$$
\begin{aligned}
& \text { I. Para } r= \pm 0,5 \text { EXCELENTE } \\
& \text { II. Para } r= \pm 0,8 \text { CONVENIENTE } \\
& \text { III. Para } r= \pm 1,0 \text { ACEPTABLE } \\
& \text { IV. Para } r=(-1) \text { a }(-1,5) \text { INSUFICIENTE } \\
& \text { V. Para } r=>(+1) \text { ó } \\
& <(-1,5) \quad \text { INACEPTABLE }
\end{aligned}
$$

El procedimiento de comparar una línea granulométrica continua con otra parabólica de grado " $r$ " y la determinación posterior del coeficiente de aptitud " $C A$ " presenta un recurso simple de evaluación comparada.

La inclusión de este procedimiento en los pliegos de especificaciones técnicas procuraría un medio simple para la recepción y valoración de los agregados por medio de una escala cualitativa y cuantitativa. 


\section{ANEJO I}

\subsection{Consideraciones sobre las funciones continuas lineal, de Ross y de Bolomey}

Visto las consideraciones desarrolladas para composiciones continuas, obteniendo una función generalizada a partir de la parábola cuadrática de GESSNER, vinculando el módulo de finura de ABRA'MS $(m)$ con el tamaño límite de la composición $\left(D, d_{n}, n\right)$ y el grado ( $r$ ) de la función, de tal modo que se obtuvo las ecuaciones (IX) y (X) que se pueden resumir en:

$$
\begin{aligned}
m_{\mathrm{G}}=n- & \left(1-A^{\tilde{n}}\right) /(1-A) \quad \operatorname{con} \tilde{n}=n \text { ó } n^{\prime} \\
= & m_{\mathrm{p}} \text { para } r \neq 1
\end{aligned}
$$

en el caso particular de la función LINEAL resulta el módulo igual a:

$m_{\mathrm{L}}=m_{\mathrm{G}}$ con la cond:ción especial de $r=1$, es decir que $A=1 / a=1 / b^{\mathrm{r}}=1 / 2=0,5$.

Los valores de " $A$ " resultan ser para los distintos casos límites los siguientes:

$$
\begin{array}{llll}
\text { Línea A } & C A=+1 & r=0,666 & A=0,630 \\
\text { Línea G } & C A=0 & r=0,500 & A=0,707 \\
\text { Línea B } & C A=-1 & r=0,333 & A=0,794 \\
\text { Línea } & C A=-1,5 & r=0,250 & A=0,841 \\
\text { Línea C } & C A=-1,6 & r=0,222 & A=0,857
\end{array}
$$

\subsection{La función de ROSS}

La ecuación (IV) que expresa la misma, evidencia a ésta como la media aritmética entre la función LINEAL y la parábola de GESSNER:

$$
m=S_{1, \mathrm{n}} \% R / 100
$$

resulta entonces:

$$
\begin{aligned}
& m_{\mathrm{R}}=n-1 / 2 \cdot\left[S_{1, \mathrm{n}}\left(d_{\mathrm{i}} / D\right)+S_{1, \mathrm{n}}\left(d_{\mathrm{i}} / D\right)^{\mathrm{0}, 5}\right]= \\
& =1 / 2 \cdot\left\{\left[\mathrm{n}-S_{1, \mathrm{n}}\left(d_{\mathrm{i}} / D\right)\right]+\left[n-S_{1, \mathrm{n}}\left(d_{\mathrm{i}} / D\right)^{0,5}\right]\right\}
\end{aligned}
$$

Por consiguiente tendremos:

$$
m_{\mathrm{R}}=1 / 2 \cdot\left(m_{\mathrm{L}}+m_{\mathrm{G}}\right)
$$

\subsection{Las funciones de BOLOMEY}

Quedan expresadas mediante la ecuación (IiI) tal que:

$$
\begin{aligned}
\% R_{\mathrm{i}} / 100 & =1-\left[(1-\mathrm{B})+B \cdot\left(d_{\mathrm{i}} / D\right)^{0,5}\right]= \\
& =1-\left[(1-B)+B \cdot a^{(\mathrm{i}-\mathrm{n})}\right]=B \cdot\left(1-a^{(\mathrm{i}-\mathrm{n})}\right)
\end{aligned}
$$


resulta entonces:

$$
m_{\mathrm{B}}=B \cdot S_{1, \mathrm{n}}\left(1-a^{(\mathrm{i}-\mathrm{n})}\right)=B \cdot\left(n-a^{-\mathrm{n}} \cdot S_{1, \mathrm{n}} a^{\mathrm{i}}\right)=B \cdot m_{\mathrm{G}}
$$

es decir que el módulo de la función de BOLOMEY resulta directamente proporcional al módulo de la función de GESSNER en relación al coeficiente $B=1-(0,10) \cdot f \cdot k$ teniendo presente que $m_{\mathrm{B}}$ incluye el cemento, de modo que para la consideración de la función para el agregado solamente, contenido en la proporción de " $\alpha_{\mathrm{p}}$ " partes en peso respecto al cemento, se tiene:

$$
m_{\mathrm{B}(\mathrm{S} / \mathrm{Cem})}=\frac{1+\alpha_{\mathrm{p}}}{\alpha_{\mathrm{p}}} \cdot m_{\mathrm{G}} \cdot B
$$

estableciendo:

$$
\alpha_{\mathrm{p}}=I_{\mathrm{p}} / C_{\mathrm{p}}=I_{\mathrm{va}} \cdot P_{\mathrm{uI}} / C_{\mathrm{va}} \cdot P_{\mathrm{uC}}=\left(P_{\mathrm{uI}} / P_{\mathrm{uC}}\right) \cdot \alpha_{\mathrm{a}}
$$

quedando para

$$
\begin{gathered}
P_{\mathrm{uI}} / P_{\mathrm{uC}}=1,22=1 / 0,82 \\
\frac{1+\alpha_{\mathrm{p}}}{\alpha_{\mathrm{p}}}=\frac{1+1,22 \cdot \alpha_{\mathrm{a}}}{1,22 \cdot \alpha_{\mathrm{a}}}=\frac{0,82+\alpha_{\mathrm{a}}}{\alpha_{\mathrm{a}}}
\end{gathered}
$$

expresión que para $\alpha_{a}=5$ a 6 varía de 1,164 a 1,136 con un valor medio de $\alpha_{a}=1,15=$ $=1 / 0,87$; mientras que el coeficiente de BOLOMEY " $B$ " varía entre los siguientes valores:

$$
\begin{aligned}
& B_{\max }=1-0,10 \times 0,8 \times 1,0=0,920 \\
& B_{\min }=1-0,10 \times 1,2 \times 1,2=0,856
\end{aligned}
$$

como término medio se puede considerar $B=$ aprox. 0,888 .

Finalmente queda la siguiente expresión:

$$
m_{\mathrm{B}(\mathrm{S} / \mathrm{Cem})}=\frac{1+1,22 \cdot \alpha_{\mathrm{a}}}{1,22 \cdot \alpha_{\mathrm{a}}} \cdot B \cdot m_{\mathrm{G}}
$$

y sustituyendo:

$$
m_{\mathrm{B}(\mathrm{S} / \mathrm{Cem})}=\frac{1}{0,87} \cdot 0,888 \cdot m_{\mathrm{G}}
$$

es decir que, término medio, no hay una diferencia apreciable entre los valores de los módulos de la función de GESSNER y la de BOLOMEY corregida sin cemento.

46 


\section{BIBLIOGRAFIA FUNDAMENTAL}

- J. A. Castiñeiras: Techología del hormigón. Palumbo, Buenos Aires, 1941.

- A. Hummel: Prontuario del hormigón. Ed. Técnicos, Barcelona, 1966.

- Betontaschenbuch. Band 1 Betontechnologie, V.E.B., Berlín, 1977.

- Jean Franquin: Los áridos en el hormigón. Ed. Técn., Barcelona, 1967.

- M. Venuat y M. Papadakis: C. y E. de cementos, morteros y hormigones. Ediciones Ulmo, Bilbaj, 1966.

- E. G. R. Petrucci, E. Globo: Concreto de cimento portland, P. Al., 1978.

- J. Bonzel, H. Bub y P. Funk: Erläuterungen zu den S.B.B. DIN 1045, W. E. \& S., Berlin, 1972,1 Band 7. Auflage. 